\title{
Opportunistic Channel Access Algorithm Based on Hidden Semi Markov Model for Cognitive Radio Networks
}

\author{
B. Senthil Kumar and Dr.S.K. Srivatsa
}

\begin{abstract}
Future generation cellular networks highly depend on Cognitive radio due to its capability to handle the primary user channel utilization. However, channel sensing and allocation for secondary user are the major factors to be considered. Several research works have been carried out to deal with the sensing problem. But, most of the existing algorithms results in high computational complexity in real time implementation. In recent years, Hidden Markov Model(HMM) has been considered as a powerful statistical tool for modeling generative sequences that can be characterised by an underlying process generating an observable sequence algorithms. Thus, based on the motivation of the earlier works carried out in HMM, this paper proposed a probability based channel sensing algorithm. Hidden markov model is used for probability calculation of primary user state and the predicted channel is validated using the proposed quality estimation method. The performance metrics used to evaluate the proposed algorithm is linear mean square error value and the channel is estimated using different estimators. The comparison of the proposed algorithm with the existing algorithms and performance of the proposed algorithm is better than the other algorithms.
\end{abstract}

Keywords--- Primary User, Secondary User, Hidden Semi Markov Model, Spectrum Sensing

\section{INTRODUCTION}

$\mathrm{I}^{\mathrm{N}}$ cognitive radio networks, channel/spectrum sensing is one of the key tasks to be performed in order to limit the interference inflicted on the primary users. Due to the significance of this consideration, different spectrum sensing methods and dynamic spectrum access strategies have been extensively studied over the last couple of years (Zhao and Sadler, 2007; Ghasemi and Sousa, 2008). Among frequentlyused practical spectrum sensing methods such as matched filtering, energy detection, and cyclo stationary detection, energy detection is commonly preferred if prior information about the structure of the primary users' signals is not available at the cognitive secondary users (Hossain et al., 2009). It is important to note that, as common to all schemes, errors in the form of false-alarms and miss-detections occur in

B. Senthil Kumar, Research Scholar, St.Peter's University, Chennai. Email:senb2002@gmail.com

Dr.S.K. Srivatsa, Senior Professor, St.Joseph's College of Engineering, Chennai

DOI: 10.9756/BIJRCE.8098 sensing, and such errors can lead to degradation in the performance.

Additionally, wideband channel sensing algorithms were studied. For instance, the authors introduced novel wideband spectrum sensing techniques in (Quan et al., 2009). The authors in (Tan et al., 2013) studied the transmission collision between the primary and secondary users, and they optimized the transmission time of the secondary users between consecutive sensing phases to maximize the throughput. Finally, in (Tandra and Sahai, 2008) the authors analyzed the limits of channel sensing methods by analyzing the SNR-wall below which sensing performance will not improve further.

As another challenge for cognitive radios, wireless transmission medium is subject to variations over time due to mobility and/or changing environment. In such cases, practical wireless systems generally employ training methods to estimate the channel fading coefficients, albeit imperfectly. On the other hand, despite their practical significance, channel estimation methods and communication in the presence of imperfectly known channel conditions have not been the focus of majority of the studies on cognitive radio systems and their performances have been investigated in detail in the literature. Transmitting known pilot symbols is generally used as a method to obtain channel state information (Tong et al., 2004). For instance, the authors in (Abou-Faycal et al., 2005) focused on pilot symbol assisted modulation approach for transceiver design for time-varying channels, and proposed causal and noncausal estimation algorithms, and compared different algorithms by maximizing the mutual information between input and output over the spacing of pilot symbols. Further results and characterizations on pilot-symbol assisted wireless communications can be found, for instance, in (Chen and Beaulieu, 2007, Taghiyar et al., 2010). Several recent studies have addressed the channel estimation problem in cognitive radio systems as well and focused on pilot allocation strategies. For instance, by minimizing the mean-squared error (MSE) of the least squares channel estimation method, a practical pilot design method for OFDM-based cognitive radios was proposed in (Hu and $\mathrm{He}, 2010)$. The relationship between the optimal pilot pattern and the power loading was investigated in (Soubachov and Ventura, 2011) under the constraint that primary users did not experience interference above a certain threshold. However, in these studies, channel sensing errors have not been considered.

(Gao et al., 2010) addressed channel training and estimation in a multiple-antenna cognitive radio setting. In their model, cognitive users initially listen to the primary 
users' transmission in order to learn the structure of the covariance matrices of the received signals and perform receive and transmit beam forming in their own transmissions. Following this phase, the cognitive users enter into a training phase in which pilot signals are sent and the Linear Minimum Mean Square-Error (LMMSE) estimation is performed. In a related study, an approach is proposed to perform spectrum sensing and data transmission simultaneously in order to maximize both the sensing time and the throughput of the cognitive system (Stotas and Nallanathan, 2010).

In this research, to overcome the problems in the existing methods by proposing a new method called Hidden Semi Markov Model for channel sensing and the predicted channel quality is estimated. The channel quality estimated using Linear Minimum Mean Square Error (LMMSE) estimator. The selected channel is used for data transmission between the secondary channels. The proposed method is explained briefly in the following sections.

\section{SYSTEM MODEL}

The system model of cognitive radio networks for sensing the channel is considered in this research in (Jan Bulla, 2006). The scenario considered in this research consist of single primary transmitter, single cognitive radio are used for operating on single narrowband channel. The primary transmitter is active only when it has data to transmit. The primary transmitter state is identified using the cognitive radio which acts as a sensor. The primary transmitter is located in the transmission range of the cognitive radio and their locations are fixed. The licensed channel does not properly utilize by the primary user as a result the channel fluctuate between idle and active states. The channel state is represented using the finite set $X=\{1 ; \ldots ; d\}$.

\section{METHODOLOGY}

\subsection{Hidden Semi Markov Model}

A hidden semi markov model is an extension of hidden markov model using the semi markov chain process with parameters such as variable duration or sojourn time for each state. In HSMM number of observation for each state is high than the number of observations present in the HMM (Sadough and Jaffrot, 2005; Sadough et al. 2009). This is the main dissimilarity between the HMM and HSMM. In this research, hidden semi markov model is used for channel sensing. The HSMM consist of pair of discrete-time stochastic processes $\left\{S_{t}\right\}$ and $\left\{X_{t}\right\}, t \in\{0, \ldots, \tau-1\}$. The observed process $\left\{X_{t}\right\}$ is linked to the hidden, i.e., unobserved state process $\left\{S_{t}\right\}$ by the conditional distribution depending on the state process.

$$
b_{j}\left(x_{t}\right)=P\left(X_{t}=x_{t} \mid S_{t}=j\right) \text { with } \sum_{x_{t}} b_{j}\left(x_{t}\right)=1
$$

\subsection{Semi-Markov Chains}

The semi markov chain process is constructed using the embedded first order markov chain and the parameters is defined by the initial probabilities $\pi_{\mathrm{i}}:=\mathrm{P}\left(\mathrm{S}_{0}=\mathrm{i}\right)$ with $\sum_{\mathrm{i}} \pi_{\mathrm{i}}=1$ and the transition probabilities for the state i. For each $\mathrm{j} \neq 1$

$$
\begin{gathered}
\mathrm{p}_{\mathrm{ij}}:=\mathrm{P}\left(\mathrm{S}_{\mathrm{t}+1}=\mathrm{j} \mid \mathrm{S}_{\mathrm{t}+1} \neq \mathrm{i}, \mathrm{S}_{\mathrm{t}}=\mathrm{i}\right) \text { with } \sum_{\mathrm{i} \neq 1} \mathrm{p}_{\mathrm{ij}}=1 \text { and } \\
\mathrm{p}_{\mathrm{ij}}=0
\end{gathered}
$$

The dwell time distributions are allotted to the states in the model in order to construct the semi-markov chain model. It is explained as follows

$$
d_{j}(u):=P\left(S_{t+u+1} \neq j, S_{t+u-v}=j, v=0, \ldots, u-2 \mid S_{t+1}=j, S_{t} \neq j\right)
$$

Where $d_{j}(u)$ is dwell time or sojourn time distribution for $\mathrm{u} \in\left\{1, \ldots, \mathrm{M}_{\mathrm{j}}\right\}$ from $\mathrm{t}+1$ to $\mathrm{t}+\mathrm{u} . \mathrm{u}$ is represented as unobserved process of length, $\mathrm{M}_{\mathrm{j}}$ is upper bound of the time spent in state j. For the calculation of sojourn time, by assuming that the state occupancy distribution is used the finite set of points $\left\{1 \ldots, \mathrm{M}_{\mathrm{j}}\right\}$, and the point $\mathrm{M}_{\mathrm{j}}$ is increased up to entire length of observed sequence. If it is last state means then the sojourn time is calculated for that particular state is

$$
\mathrm{D}_{\mathrm{j}}(\mathrm{u}):=\sum_{\mathrm{v} \geq \mathrm{u}} \mathrm{d}_{\mathrm{j}}(\mathrm{v})
$$

It is also called as survivor function of the sojourn time in state $\mathrm{j}$. The survivor function is defined as a mean of individual probability masses of all possible sojourns of length $\mathrm{v} \geq \mathrm{u}$. The combination of first order markov chain and state occupancy distribution is used for the construction a semiMarkov chain. If the process starts in state $\mathrm{j}$ at time $\mathrm{t}=0$, the following relation can be verified.

$$
P\left(S_{t} \neq j, S_{t-v}=j, v=1, \ldots, t\right)=d_{j}(t) \pi_{j}
$$

\subsection{Parameter Estimation}

The HSMM based on HDP denoted by $\phi=(\pi, \mu, H, G)$ where $\pi$ is an initial probability, a vector of mean observed signal strengths $\mu$, a transition matrix $H$, a vector of observed signal strength variances $G$. The parameter estimation of hidden semi markov model is more complicated than the hidden markov model (Sadough, 2008). The EM algorithm is used for calculating the unknown parameters of the hidden semi markov model from real data in the maximum likelihood sense. Given an initial parameter estimate $\phi$ and a sequence of signal strength observations $Y_{t}, t=\{0, \ldots, T\}$ a parameter estimate $\hat{\phi}$ with higher likelihood is computed. The steps used to calculate the parameter estimation is explained as follows

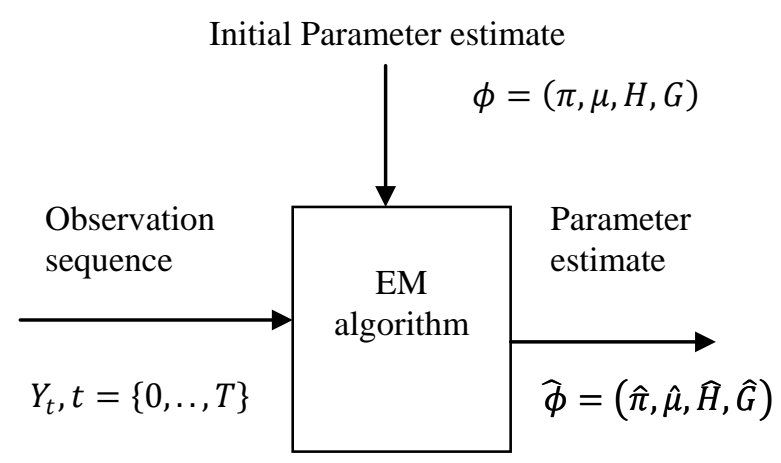

Figure 1: Parameter Estimation using Baum Algorithm 
The E-step involves estimating two terms: 1) the probability of the current state is calculated using the below formula

$\mathrm{p}\left(\mathrm{z}_{\mathrm{t}-1}, \mathrm{z}_{\mathrm{t}} \mid \mathrm{y}_{0}^{\mathrm{T}} ; \phi\right), \mathrm{t}=1, \ldots, \mathrm{T}$ which can be efficiently calculated as follows:

$$
\begin{aligned}
& \mathrm{p}\left(\mathrm{z}_{\mathrm{t}-1}, \mathrm{z}_{\mathrm{t}} \mid \mathrm{y}_{0}^{\mathrm{T}} ; \phi\right) \\
& =\frac{\bar{\alpha}\left(\mathrm{z}_{\mathrm{t}-1} \mathrm{y}_{0}^{\mathrm{t}-1}\right) \bar{\beta}\left(\mathrm{y}_{\mathrm{t}+1}^{\mathrm{T}} \mid \mathrm{z}_{\mathrm{t}}\right)[\mathrm{H}]_{\mathrm{z}_{\mathrm{t}-1}, \mathrm{z}_{\mathrm{t}}} \mathrm{p}\left(\mathrm{y}_{\mathrm{t}} \mid \mathrm{x}_{\mathrm{t}}\right)}{\sum_{\mathrm{z}_{\mathrm{t}-1,1}, \mathrm{z}_{\mathrm{t}}} \bar{\alpha}\left(\mathrm{z}_{\mathrm{t}-1} \mathrm{y}_{0}^{\mathrm{t}-1}\right) \bar{\beta}\left(\mathrm{y}_{\mathrm{t}+1}^{\mathrm{T}} \mid \mathrm{z}_{\mathrm{t}}\right)[\mathrm{H}]_{\mathrm{z}_{\mathrm{t}-1, \mathrm{z}_{\mathrm{t}}}} \mathrm{p}\left(\mathrm{y}_{\mathrm{t}} \mid \mathrm{x}_{\mathrm{t}}\right)}
\end{aligned}
$$

Where $[\mathrm{H}]_{\mathrm{z}_{\mathrm{t}-1}, \mathrm{z}_{\mathrm{t}}}$ denotes the $\left(\mathrm{z}_{\mathrm{t}-1}, \mathrm{z}_{\mathrm{t}}\right)$ entry of the transition matrix $\mathrm{H}$.

1) The probability of the process left state and entered state is calculated given below

$$
\xi_{\mathrm{t}}(\mathrm{i}, \mathrm{j})=\mathrm{P}\left(\mathrm{z}_{\mathrm{t}}=\mathrm{i}, \mathrm{z}_{\mathrm{t}+1}=\mathrm{j} \mid \mathrm{X}=\mathrm{x} ; \theta\right)
$$

2) The expected number of times a process spends $u$ time steps in state $\mathbf{j}$

$$
\begin{aligned}
\eta_{i u}=P\left(z_{u} \neq i, z_{u-v}=i, v=1, \ldots, u \mid X=x ; \theta\right) \\
+\sum_{\substack{t=1 \\
=}} P\left(z_{t+u+1} \neq i, z_{t+u-v}=i, v\right. \\
\left.0, \ldots, u-1, S_{t} \neq i \mid X=x ; \theta\right)
\end{aligned}
$$

as

M steps: 1) the initial transition probabilities are estimated

$$
\begin{gathered}
\hat{\pi}_{\mathrm{j}, \mathrm{i}}=\mathrm{p}\left(\mathrm{z}_{0}=(\mathrm{a}, \mathrm{i}) \mid \mathrm{y}_{0}^{\mathrm{T}} ; \phi\right) \\
\hat{\mathrm{h}}_{\mathrm{ab}}(\mathrm{ij})=\frac{\sum_{\mathrm{t}=1}^{\mathrm{T}} \mathrm{p}\left(\mathrm{z}_{\mathrm{t}-1}=(\mathrm{a}, \mathrm{i}), \mathrm{z}_{\mathrm{t}}=(\mathrm{b}, \mathrm{j}) \mid \mathrm{y}_{0}^{\mathrm{T}} ; \phi\right)}{\sum_{(\mathrm{b}, \mathrm{j}) \in \mathbb{Z}} \sum_{\mathrm{t}=1}^{\mathrm{T}} \mathrm{p}\left(\mathrm{z}_{\mathrm{t}-1}=(\mathrm{a}, \mathrm{i}), \mathrm{z}_{\mathrm{t}}=(\mathrm{b}, \mathrm{j}) \mid \mathrm{y}_{0}^{\mathrm{T}} ; \phi\right)}
\end{gathered}
$$

The aforementioned equation is mainly used for the calculation of parameters and if $X_{t}$ is considered as normally distributed ie., $X_{t} \mid S_{t}=i \sim N\left(\mu_{i}, \sigma_{i}^{2}\right)$ then parameters $\mu_{i}$ and $\sigma_{i}^{2}$ can be estimated as

$$
\begin{aligned}
& \hat{\mu}_{\mathrm{i}}=\frac{\sum_{\mathrm{t}=1}^{\mathrm{T}} \sum_{\mathrm{i}=1}^{\mathrm{r}} \mathrm{p}\left(\mathrm{z}_{\mathrm{t}}=(\mathrm{i}) \mid \mathrm{y}_{0}^{\mathrm{T}} ; \phi\right) \mathrm{y}_{\mathrm{t}}}{\sum_{\mathrm{t}=1}^{\mathrm{T}} \sum_{\mathrm{i}=1}^{\mathrm{r}} \mathrm{p}\left(\mathrm{z}_{\mathrm{t}}=(, \mathrm{i}) \mid \mathrm{y}_{0}^{\mathrm{T}} ; \phi\right)} \text { and } \\
& \hat{\sigma}_{\mathrm{i}}=\frac{\sum_{\mathrm{t}=1}^{\mathrm{T}} \sum_{\mathrm{i}=1}^{\mathrm{r}} \mathrm{p}\left(\mathrm{z}_{\mathrm{t}}=(, \mathrm{i}) \mid \mathrm{y}_{0}^{\mathrm{T}} ; \phi\right)\left(\mathrm{y}_{\mathrm{t}}-\hat{\mu}_{\mathrm{i}}\right)}{\sum_{\mathrm{t}=1}^{\mathrm{T}} \sum_{\mathrm{i}=1}^{\mathrm{r}} \mathrm{p}\left(\mathrm{z}_{\mathrm{t}}=(, \mathrm{i}) \mid \mathrm{y}_{0}^{\mathrm{T}} ; \phi\right)}
\end{aligned}
$$

3) The state duration density is estimated using the following the equation based on the non probability mass function

$$
\mathrm{d}_{\mathrm{i}}(\mathrm{u})=\frac{\eta_{\mathrm{iu}}}{\sum_{\mathrm{v}} \eta_{\mathrm{iu}}}
$$

The cognitive radio systems performance is evaluated using the aforementioned parameter evaluation by generating the simulated data. The evaluation of transition matrix $\widehat{H}$ using the parameter estimate $\hat{\phi}$ is a complex process and it is used to obtain the dynamics of the primary transmitter state process. Different propagation models and shadowing variance can be represented by adjusting $\hat{\mu}$ and $\widehat{\mathrm{G}}$ as appropriate, while retaining the same $\widehat{\mathrm{H}}$.

\subsection{State Estimation and Prediction}

Let us consider the parameter estimation of HSMM as $\phi$ like aforementioned. The primary user state is represented as $\mathrm{X}_{\mathrm{t}}$ and here two states are considered 0 and 1 (Tian and Giannakis, 2006). $X_{t}=0$ represents the idle state and $X_{t}=1$ represents active state. Then the state is estimated using the parameter of HSMM as

$$
\begin{gathered}
\mathrm{p}\left(\mathrm{z}_{\mathrm{t}+\mathrm{m}} \mid \mathrm{y}_{0}^{\mathrm{T}} ; \phi\right)=\sum_{\mathrm{z}_{\mathrm{t}}} \mathrm{p}\left(\mathrm{z}_{\mathrm{t}} \mid \mathrm{y}_{0}^{\mathrm{T}} ; \phi\right) \mathrm{p}\left(\mathrm{z}_{\mathrm{t}+\mathrm{m}} \mid \mathrm{z}_{\mathrm{t}} ; \phi\right) \\
=\sum_{\mathrm{z}_{\mathrm{t}}} \bar{\alpha}\left(\mathrm{z}_{\mathrm{t}}, \mathrm{y}_{0}^{\mathrm{T}}\right)\left[\mathrm{H}^{\mathrm{m}}\right]_{\mathrm{z}_{\mathrm{t}-1, \mathrm{z}_{\mathrm{t}}}}
\end{gathered}
$$

for $\mathrm{m} \geq 0$ and $\mathrm{t} \geq 0$. As mentioned above, the complexity of the forward recursion for computing $\bar{\alpha}\left(z_{t}, y_{0}^{t}\right)$ is $\mathrm{O}\left(\mathrm{d}^{2} \mathrm{r}^{2}\right)$ per step. Since $\mathrm{H}^{\mathrm{m}}$ can be pre-computed, the computational complexity of the forward recursion is also $\mathrm{O}\left(\mathrm{d}^{2} \mathrm{r}^{2}\right)$, or $\mathrm{O}\left(\mathrm{r}^{2}\right)$ when $\mathrm{d}=2$. A detection scheme for the state process of the semi Markov chain at time $t+m$, given $y_{0}^{t}$ , is obtained from

$$
\widehat{\mathrm{X}}_{\mathrm{t}+\mathrm{m} \mid \mathrm{t}}=\left\{\begin{array}{rr}
0, \text { if } \mathrm{p}\left(\mathrm{x}_{\mathrm{t}+\mathrm{m}}=1 \mid \mathrm{y}_{0}^{\mathrm{T}} ; \phi\right) \geq \gamma \\
1, & \text { otherwise }
\end{array}\right.
$$

where using (22) we can calculate

$$
\mathrm{p}\left(\mathrm{x}_{\mathrm{t}+\mathrm{m}}=1 \mid \mathrm{y}_{0}^{\mathrm{T}} ; \phi\right)=\sum_{\mathrm{s}_{\mathrm{t}+\mathrm{m}}} \mathrm{p}\left(\mathrm{z}_{\mathrm{t}+\mathrm{m}}=\left(1, \mathrm{~s}_{\mathrm{t}+\mathrm{m}}\right) \mid \mathrm{y}_{0}^{\mathrm{T}} ; \phi\right)
$$

And $0<\gamma<1$ is a decision threshold. The computational complexity of the detector given by (23) consists of $\mathrm{O}\left(\mathrm{r}^{2}\right)$ multiplications.

Based on the above the calculation the primary user state is estimated using the hidden semi markov model probability and if the channel is idle state then the channel is selected for further data transmission. After the channel is selected it is verified using the channel quality prediction based on the mathematical formulation mentioned below. Selected channel is given as a input to the channel quality prediction.

\subsection{Sensed Channel Quality Prediction}

The selected channel based on the state prediction of primary user is given as input to the quality prediction and it is evaluated using two metrics. The first parameter for channel quality prediction used here is primary channel sensing accuracy of the secondary user and the second parameter is duration of the channel availability. In this research, the channel is sensed using the probability of HSMM. The first parameter channel sensing accuracy can be calculated using the higher detection probability $\mathrm{P}_{\mathrm{d}}$ and false alarm probability $\mathrm{P}_{\mathrm{f}}$. The general formula for the calculation of

$$
\mathrm{C}_{\mathrm{sa}}=\mathrm{P}_{\mathrm{d}}\left(1-\mathrm{P}_{\mathrm{f}}\right)
$$

The second parameter is calculated using formula given below

$$
\mathrm{C}_{\mathrm{id}}=1 / \lambda
$$

Where $\lambda$ is estimated duration of the primary state. The channel quality is calculated by combining the two parameters and the formula for the channel quality prediction is given below 


$$
\mathrm{C}_{\mathrm{q}}=\left(1+\log _{\epsilon} \mathrm{C}_{\mathrm{sa}}\right) \mathrm{C}_{\mathrm{id}}
$$

Where $\epsilon$ is used to represent the preference of the secondary channel and it can be derived as follows

$$
\begin{gathered}
\frac{\partial \mathrm{C}_{\mathrm{Q}}}{\partial \mathrm{C}_{\mathrm{id}} \partial \epsilon}=-\ln \mathrm{C}_{\mathrm{sa}}\left(\frac{1}{\ln \epsilon}\right)^{2}>0 \\
\frac{\partial \mathrm{C}_{\mathrm{Q}}}{\partial \mathrm{C}_{\mathrm{id}} \partial \epsilon}=-\frac{\mathrm{C}_{\mathrm{sa}}}{\mathrm{C}_{\mathrm{id}} \epsilon}\left(\frac{1}{\ln \epsilon}\right)^{2}<0
\end{gathered}
$$

For the second parameter the channel idle duration $\epsilon$ value should be high and for first parameter the channel sensing accuracy $\epsilon$ value should be low.

\section{EXPERIMENTAL RESULTS}

The simulation results of proposed sensing algorithm are present in this section. the linear minimum mean-square errors (LMMSEs) of the proposed HSMM spectrum sensing algorithm is compared with the existing algorithms such spectrum sensing based on random scheduling and optimal scheduling based spectrum sensing. The selected channel is validated using the estimator such as LMMSEs and proposed channel quality prediction in order to measure errors. The parameter used for experimental results comparison is false alarm probability, detection probability and it is shown in the table 1 .

Table 1: Parameter Used in Experimental Results

\begin{tabular}{|l|l|}
\hline Parameter & value \\
\hline Length of the state /observation sequence (T) & 100 \\
\hline False alarm probability & {$[0.01,0.1]$} \\
\hline Detection probability & {$[0.09,0.99]$} \\
\hline
\end{tabular}

\section{Linear MMSE Estimation}

The LMMSEs values are estimated from this following procedure. Let us assume that have to estimate a scalar parameter based on the data set $\{x[0], x[l], \ldots, x[N-1]\}$ or in vector form $X=[x[0] x[l] \ldots x[N-I]]^{T}$. Do not assume any specific knowledge of the joint $\operatorname{PDF} p(x, \theta)$, because as, knowledge of the first two moments suffices. Show that the correlation between $\theta$ and $\mathrm{X}$ is sufficient to estimate. Then consider the class of all linear estimators of the form

$$
\hat{\theta}=\sum_{n=0}^{N-1} a_{n} x[n]+a_{N}
$$

The task is to choose the weighting coefficients $a_{N}$ to minimize the Bayesian MSE (Mean Square Error)

$$
\operatorname{Bmse}(\hat{\theta})=E\left[(\theta-\hat{\theta})^{2}\right]
$$

Here the expectation is with respect to the $\operatorname{PDF} p(x, \theta)$. The resultant estimator is termed the linear minimum mean square error (LMMSE) estimator. Here the term $a_{N}$ has been incorporated to allow for a mismatch between the means of $\theta$ and $\mathrm{x}$. If the means are equal then this coefficient is zero and may be omitted.

\subsection{Noise Variance}

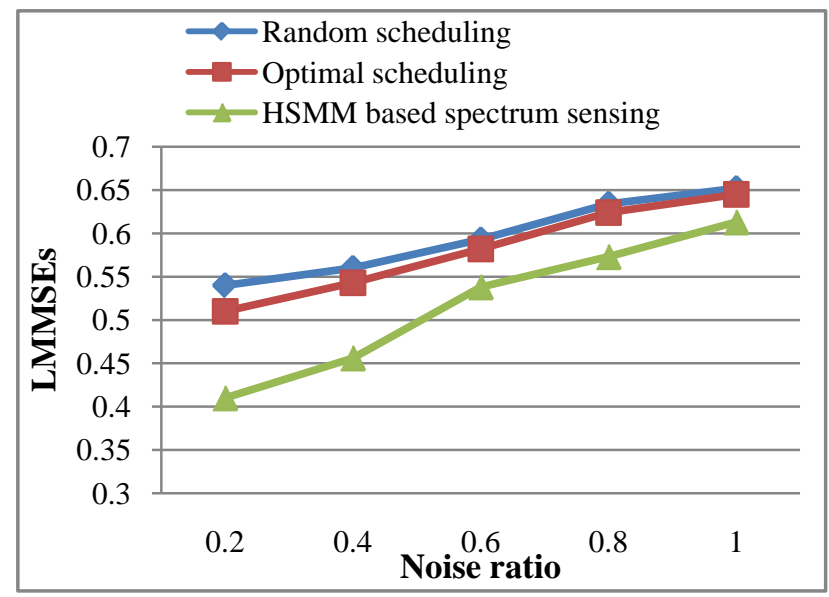

Figure 2: LMMSE vs. Average Noise Power

In Fig. 2, the linear minimum mean-square errors (LMMSEs) of the proposed HSMM spectrum sensing algorithm is compared with the existing algorithms such spectrum sensing based on random scheduling and optimal scheduling based spectrum sensing. It is observed that the proposed algorithms achieve the lowest LMMSEs whereas other algorithms had the worst performance, as expected. In addition, as the noise variance increases, the LMMSEs increase and the performance of the estimators of the proposed approach obtained nearer to each other. The table 2 shows that the values of the comparison of LMMSE vs noise power for proposed and existing algorithms.

Table 2: Comparison of LMMSE vs. Noise Power

\begin{tabular}{|l|l|l|l|}
\hline $\begin{array}{c}\text { Noise } \\
\text { power }\end{array}$ & $\begin{array}{c}\text { Random } \\
\text { scheduling }\end{array}$ & $\begin{array}{c}\text { Optimal } \\
\text { scheduling }\end{array}$ & $\begin{array}{c}\text { HSMM based } \\
\text { spectrum sensing }\end{array}$ \\
\hline 0.2 & 0.54 & 0.51 & 0.41 \\
\hline 0.4 & 0.56 & 0.543 & 0.536 \\
\hline 0.6 & 0.593 & 0.582 & 0.568 \\
\hline 0.8 & 0.634 & 0.624 & 0.593 \\
\hline 1 & 0.652 & 0.645 & 0.613 \\
\hline
\end{tabular}

\subsection{Detection Probability}

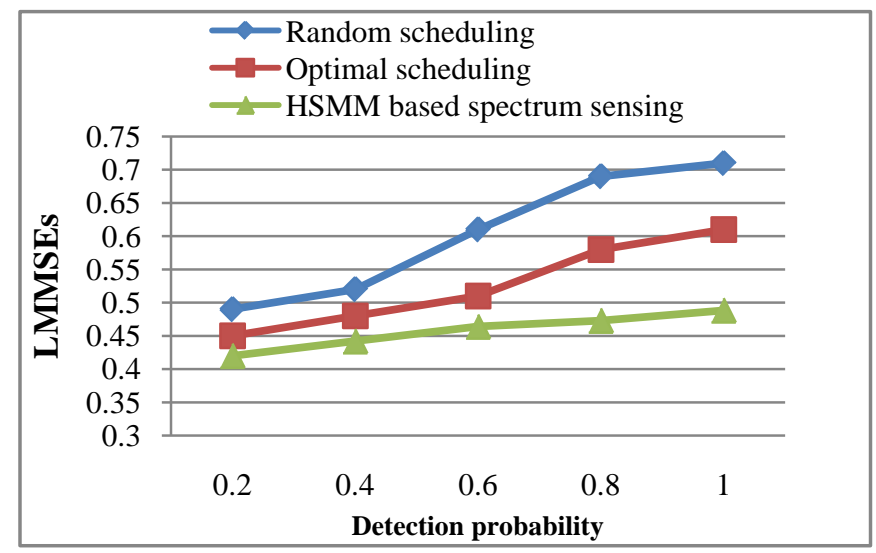

Figure 3: Comparison of LMMSE vs. Detection Probability

In Fig. 3, the linear minimum mean-square errors (LMMSEs) of the proposed HSMM spectrum sensing algorithm (Senthil Kumar and Srivatsa , 2014) is compared with the existing algorithms such spectrum sensing based on 
random scheduling and optimal scheduling based spectrum sensing. It is observed that the proposed algorithms achieve the lowest LMMSEs whereas other algorithms had the worst performance, as expected. Table 3 shows that the values of the comparison of LMMSE vs. detection probability for proposed and existing algorithms.

Table 3: Comparison of LMMSE vs. Detection Probability

\begin{tabular}{|l|l|l|l|}
\hline $\begin{array}{l}\text { Detection } \\
\text { probability }\end{array}$ & $\begin{array}{l}\text { Random } \\
\text { scheduling }\end{array}$ & $\begin{array}{l}\text { OPTIMAL } \\
\text { Scheduling }\end{array}$ & $\begin{array}{l}\text { HSMM based } \\
\text { spectrum } \\
\text { sensing }\end{array}$ \\
\hline 0.5 & 0.49 & 0.45 & 0.413 \\
\hline 0.6 & 0.52 & 0.48 & 0.4386 \\
\hline 0.7 & 0.61 & 0.51 & 0.4432 \\
\hline 0.8 & 0.69 & 0.58 & 0.4516 \\
\hline 0.9 & 0.71 & 0.61 & 0.4594 \\
\hline 1 & 0.79 & 0.67 & 0.4612 \\
\hline
\end{tabular}

\subsection{False Alarm Probability}

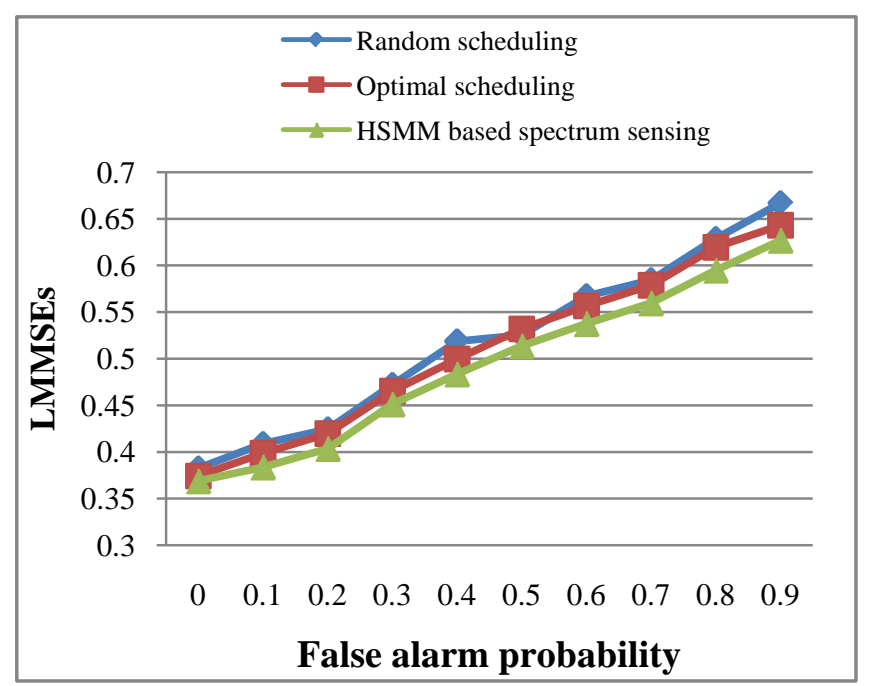

Figure 4: Comparison of LMMSE vs. False Alarm Probability

In Fig. 4, the linear minimum mean-square Errors (LMMSEs) of the proposed HSMM spectrum sensing algorithm is compared with the existing algorithms such spectrum sensing based on random scheduling and optimal scheduling based spectrum sensing. The false alarms for proposed secure spectrum sensing are almost same when the fraction of the malicious nodes is less than 0.63 . The figure also shows that the false alarm probability of existing methods is much higher than those of proposed schemes.

Table 4 comparison of LMMSE vs. false alarm probability

\begin{tabular}{|l|l|l|l|}
\hline $\begin{array}{l}\text { False alarm } \\
\text { probability }\end{array}$ & $\begin{array}{l}\text { Random } \\
\text { scheduling }\end{array}$ & $\begin{array}{l}\text { Optimal } \\
\text { Scheduling }\end{array}$ & $\begin{array}{l}\text { HSMM based } \\
\text { spectrum } \\
\text { sensing }\end{array}$ \\
\hline 0 & 0.3825 & 0.3743 & 0.3689 \\
\hline 0.1 & 0.4089 & 0.3988 & 0.3838 \\
\hline 0.2 & 0.4245 & 0.4198 & 0.4037 \\
\hline 0.3 & 0.4721 & 0.4653 & 0.4514 \\
\hline 0.4 & 0.5188 & 0.4998 & 0.4836 \\
\hline 0.5 & 0.5254 & 0.5321 & 0.5137 \\
\hline 0.6 & 0.5673 & 0.5567 & 0.5376 \\
\hline 0.7 & 0.5845 & 0.5789 & 0.5598 \\
\hline 0.8 & 0.6288 & 0.6196 & 0.5949 \\
\hline 0.9 & 0.6669 & 0.6432 & 0.6269 \\
\hline
\end{tabular}

\section{CONCLUSION}

This paper presented a channel estimation algorithm using the channel sensing errors and the channel is sensed using the probability calculation of primary users using Hidden semi markov model. Once the channel is sensed then the channel quality is estimated using the proposed mathematical formulation. The performance of the selected channel is measured using the proposed estimator and different estimator such as LMMSE, Mismatched MSE. The results show that the proposed algorithm and estimator is better than the conventional algorithms.

\section{REFERENCE}

[1] Zhao Q and B. Sadler (2007), “A survey of dynamic spectrum access,” IEEE Sig. Pro. Mag., vol. 24, no. 3, pp. 79-89.

[2] Ghasemi A and E. S. Sousa (2008), "Spectrum sensing in cognitive radio networks: Requirements, challenges and design trade-offs,” IEEE Comm. Mag., vol. 46, no, 4, pp. 32-39.

[3] Hossain, E, Niyato D and Z. Han (2009), Dynamic Spectrum Access and Management in Cognitive Radio Networks, Cambridge University Press.

[4] Quan, Z Cui, S. A. H. Sayed, and H. V. Poor (2009), "Optimal multiband joint detection for spectrum sensing in cognitive radio networks,” IEEE Trans. Signal Process., vol. 57, no. 3.

[5] Tan, S.-S. J. Zeidler, and B. Rao (2013), “Opportunistic Channel-Aware Spectrum Access for Cognitive Radio Networks with Interleaved Transmission and Sensing,” IEEE Trans. Wireless Commun., vol. 12, no. 5.

[6] Tandra R. and A. Sahai (2008), "SNR walls for signal detection," IEEE Journal of Selected Topics in Signal Processing, vol. 2, no. 1, pp. 4-17, Feb. 2008.

[7] Tong, L. B. M. Sadler and M. Dong 2004, "Pilot-assited wireless transmissions,” IEEE Signal Process. Mag.

[8] Abou-Faycal, I M. M'edard, and U. Madhow (2005), "Binary adaptive coded pilot symbol assisted modulation over Rayleigh fading channels without feedback,” IEEE Trans. Commun., vol. 53, no. 6.

[9] Chen Y. and N. C. Beaulieu (2007), "Optimum pilot symbol assisted modulation,” IEEE Trans. Commun., vol. 55, no. 8

[10] Taghiyar, M. J. S. Muhaidat, and J. Liang, "On the performance of pilot symbol assisted modulation for cooperative systems with imperfect channel estimation,” Proc. of IEEE Wireless Communications and Networking Conference (WCNC), April 18-21, 2010.

[11] Hu D. and L. He (2010), "Pilot design for channel estimation in OFDMbased cognitive radio systems," Proc. of IEEE Global Telecommunications Conference (GLOBECOM), Dec. 6-10.

[12] Soubachov B. V. and N. Ventura (2011), "Optimal pilot placement in cognitive radio systems for Wiener filtered MMSE channel estimation,” Proc. of IARIA Conference on Advances in Cognitive Radio, April 1722.

[13] Gao, F. R. Zhang, Y.-C. Liang, and X. Wang (2010), "Design of learning-based MIMO cognitive radio systems," IEEE Trans. Vehicular Technology, vol. 59, no. 4., pp. 1707-1720.

[14] Stotas S. and A. Nallanathan (2010), "Overcoming the sensingthroughput tradeoff in cognitive radio networks,” Proc. IEEE Int. Conf. on Commun., Cape Town.

[15] Jan Bulla, (2006), "Application of Hidden Markov Models and Hidden Semi-Markov Models to Financial Time Series”.

[16] Sadough SMS and E. Jaffrot (2005), "A wavelet packet based model for an ultra-wideband propagation channel," in Proc. European Conference on Propagation and Systems (ECPS).

[17] Sadough SMS, M. M. Ichir, P. Duhamel, and E. Jaffrot (2009), "Waveletbased semiblind channel estimation for ultrawideband OFDM systems,” IEEE Trans. Vehicular Technology, no. 3, pp. 1302-1314.

[18] Sadough (2008), "Ultrawideband OFDM channel estimation through a wavelet based EM-MAP algorithm," European Transactions on Telecommunications,pp. 761-771.

[19] B. Senthil Kumar and S.K. Srivatsa," An Efficient Channel Sensing Algorithm Based on Hidden Semi Markov Model and Channel Quality Prediction", Research Journal of Applied Sciences, Engineering and Technology 8(19): 2064-2070, 2014 